\title{
A-B Autonomy of A Shape-shifting Robot "AMOEBA-I" for USAR
}

\author{
Yuechao Wang, Jinguo Liu and Bin Li \\ State Key Laboratory of Robotics \\ Shenyang Institute of Automation \\ Chinese Academy of Sciences, P.R. China
}

\section{Introduction}

The frequent nature disasters and man-made catastrophes during the last decades such as earthquake, typhoon, hurricane, radiation and terrorist attack have aroused people's attention on the importance of Urban Search and Rescue (USAR). Although people have more watchfulness than before, a large number of people still have died in unprofessional rescue due to inadequate equipment and being lack of professional manpower (Erkmen et al, 2002; Casper and Murphy, 2003). Timely searching for victims and subsequent rescue operations from the rubble of collapsed buildings are highly required. Since these operations are very dangerous for human workers and even for trained dogs, autonomous mobile systems are highly needed to help in finding trapped victims. It is a great challenge to develop search and rescue robot that can actually work in the disaster site. The search and rescue robot research includes not only the robotics technology but also the rescue technology and the disaster science. Researches sponsored by the governments and the companies have resulted in the emergence of various kinds of search and rescue robots. Since earthquake happens in Japan frequently, intelligent rescue systems and robotic technology have been expected to mitigate disaster damages, especially after the 1995 Hanshin-Awaji Earthquake. From 2002, "Special Project for Earthquake Disaster Mitigation in Urban Areas" (a 5 years project which also called DDT project) was launched by Ministry of Education, Culture, Sports, Science and Technology, in Japan. Many kinds of search and rescue robots have been developed (Matsuno and Tadokoro, 2004), such as Souryu (Hirose and Fukushima, 2002), Moira (Osuka and Kitajima, 2003), and KOHGA (Tetsushi et al, 2004). In America the rescue robot research has attracted a lot attention too. Several robots were used for the search and detection operation in the collapsed World Trade Center building in September 2001 (Casper and Murphy, 2003). In the University of South Florida, Professor Murphy and her fellow have developed "Bujold", a kind of search and rescue robot that has the ability of shape shifting and has been equipped with many sensors (Murphy, 2002). In Carnegie Mellon Robotics Institute, researchers have developed multi-joint robot for inspection (Wolf et al, 2003). Foster-Miller Company also carries out TALON Robot series for search and rescue mission. In China, a series of search and rescue robots have been developed in recent years (Liu et al, 2007a; Zhang et al, 2006; Zou et al, 
2006; Huang et al, 2006). Among them a shape-shifting robot named "AMOEBA-I" has been developed in the Shenyang Institute of Automation, CAS for search and rescue operation (Liu et al, 2004; Li et al, 2006). Since search and rescue robots are usually unmanned vehicles, autonomy or autonomous behaviours play key roles in their performances. In this chapter, we first provide a brief description of AMOEBA-I. Then, A-B Autonomy of "AMOEBA-I" has been discussed. Rules of autonomous shape-shifting for AMOEBA-I in urban terrain have been proposed according to the features of AMOEBA-I. Finally, experimental results from its autonomous motions (autonomous shape-shifting, autonomous navigation, autonomous climbing up obstacle and autonomous avoiding obstacle) have demonstrated that the AMOEBA-I shows great autonomy in urban terrain.

\section{Mechanism of shape-shifting}

In unstructured environment operations such as search and rescue, flexibility and adaptability are urgently required in such situations for mobile robots since the terrain is usually uneven and unpredictable. In nature, there are many kinds of creatures with high motilities. For instance, the link-type structure animals, such as snakes and centipedes, behave perfect mobility and flexibility in uneven terrain for their redundancy. The link-type structures also widely exist in snake-like robots, serpentine robots, and multi-joint robots. Inspired by the nature snake that has perfect mobility under various environments, link-type robots have been widely studied. Link-type structures are connected sequentially with active or passive joints between the adjacent units or modules. They have a large number of degrees of freedom. Powered links between the units allow the chain to adapt to the terrain, to adopt the desired shape for a particular task, and to lift the front modules to surmount obstacles. Generally, the link-type structures, which have been widely applied in snake-like robot, train-like robot, trailer-like robot, and multi-joint robot, have turned out to be effective mobile mechanisms for reasons listed as follows:

(1) A link-type robot with active body has excellent mobility. It can pass through narrow spaces in line as shown in Figure 1 (a), cross a ditch by stiffening the body to bridge the ditch as shown in Figure 1 (b), and lift the front part of the body to overcome the obstacles as shown in Figure 1 (d).

(2) With a low barycentre and sufficient contact to the ground, it has high longitudinal stability over uneven terrain and soft ground. As shown in Figure 1 (c), it can steadily cross a marsh by loosing joints to distribute its weight to the whole body.

(3) For a link-type structure with active joints, it can adopt manifold gaits of snakes such as serpentine motion, concertina motion, side-winding motion, rectilinear motion, thrusting motion, pushing motion, and jumping under different environments.

(4) The link-type structure can be an excellent manipulator by transforming the hyper redundant degrees of freedom just like the trunk of an elephant.

(5) The link-type structure is fault-tolerant to some extent. The reliability and maintainability can be high since the rest can still finish the mission even if a link cannot work. The malfunctioning segment can also easily be detached and replaced.

(6) The link-type structure can be easily modularized and reconfigurable. A single modular unit only has a limited mobility but a swarm of them generates better performances in many areas. 


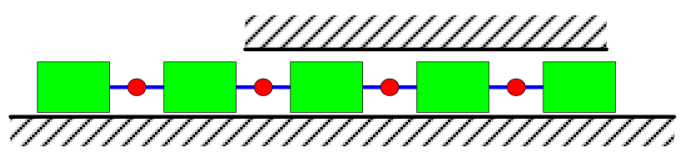

(a)

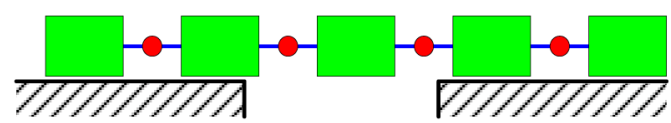

(b)

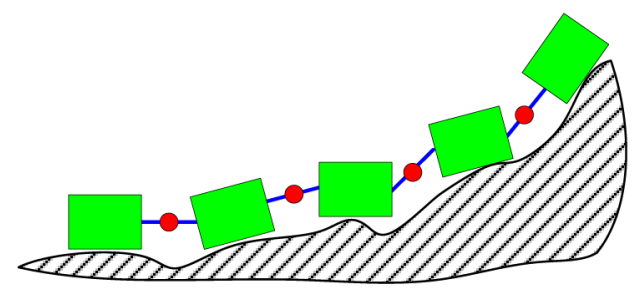

(c)

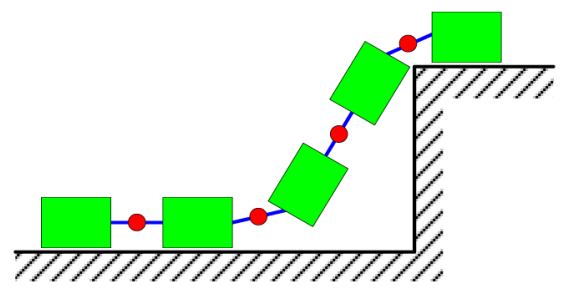

(d)

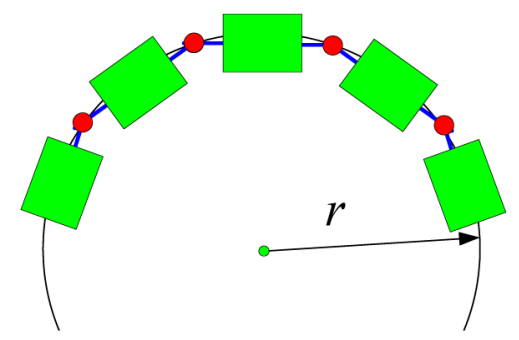

(e)

Fig. 1. Typical motilities of the link-type structure. (a) Pass through narrow space. (b) Cross a ditch. (c) Move over uneven terrain. (d) Overcome the obstacle. (e) Turn around. 
However, being long and slim, the link-type structure also has its disadvantages in some situations such as in the debris which mainly include:

(1) Lateral stability: When moving over uneven terrain, it easily gets lateral tip over as shown in Figure 1 (c). If a tip over incident happens, it may result in a series of problems: loss of traction, entrapment, system damage, loss of control, difficulty in overturning back, and even mission failure.

(2) Turning mobility: For example, when turning, it needs a large radius as shown in Figure 1 (e). When in a narrow space, it is hard for the link-type structure to turn around effectively.

How to take the advantages and surmount the disadvantages of link-type structures is both interesting and challenging tasks. For a traditional link-type structure, it can only pose the straight or curve configuration since the degree of freedom between adjacent units is usually limited by joints' movable range. Therefore, we intend to develop a self-reconfigurable link-type robot to improve the tip over stability and the turning mobility of the link-type structure. The self-reconfigurable or shape-shifting robots have attracted great attentions since they can autonomously change their physical configurations or their structure to meet the requirement of the mission and the environment. And the tracked type robots have been widely developed for search and research operation. There are usually two kinds of reconfigurable track type mobile robot in the literatures. One kind is the track's shape being changeable. Reconfigurable tracks can change from one shape to another without modification of the vehicle. This approach effectively provides various dimensions of the track contacting the ground and the track above the ground. Bujold is a typical example of this category. It is a commercially available tracked chemical inspection microrobot which can change shape among three canonical configurations: sitting up and facing forward, sitting up and facing backward, and lying flat (Murphy, 2002). A new type of variable geometry single-track driving mechanism for a rescue robot has been proposed (Lim et al, 2005). This mechanism has a symmetrical configuration so that the robot advances in dual directions and prepares against overturning. Using transformation, it can reduce the energy consumption in steering and rotating while maximizing the capacity to overcome stairs. The other kind of the reconfigurable track type robot is similar to the self-reconfigurable modular robot in principle. For instance, NUGV is a novel multi-degree-of-freedom tracked robot that can change its conformation and dimensions, and negotiate a great range of environmental dimensionality (Blackburn et al, 2004). The tracked robot CUBIC-R's shape is a regular hexahedron and each surface has a crawler unit with a couple of crawlers. As each surface is united by transformational mechanism which has 1-DOF, it can overcome rough terrains (step, stairs, gap and so on) by using transformation (Tabata et al, 2005). The reconfigurable modular type tracked robots have attracted more and more attentions for their high mobility. And they will be the ideal selections for urban or field environments. To improve the environmental adaptability of the link-type tracked robots, we have proposed a novel link-type reconfigurable structure as shown in Figure 2. This link-type structure, with Yaw and Pitch offset joints at each module's both lateral sides, has enough flexibility for its shape change. The module body is a mobile unit with active tracks. The arms and joints play important roles in shape changing process. Figure 2 demonstrates a sequence of two-module's reconfiguration. In Figure 2 (a) the link-type is in a line type while in Figure 2 (e) it is in a row type. From line-type to row type, the Yaw joint rotates $180^{\circ}$ and then so 
does the Pitch joint. It behaves the advantages of the link-type structure while in line, moreover, it can avoid the line-type's disadvantages while in row. For instance, the robot in row can turn easily with a zero radius by driving the lateral modules differentially. This link changes its configurations through a sequence of joints' rotation. Compared to reconfiguration by connection and disconnection, such kind of reconfiguration has higher reliability, less power consumption, and less time consumption.

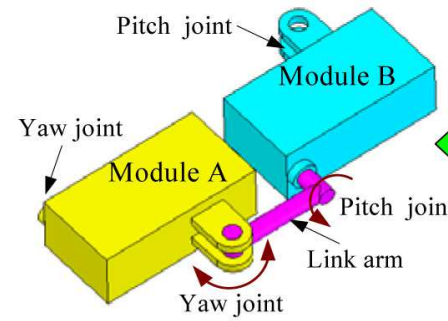

(a)

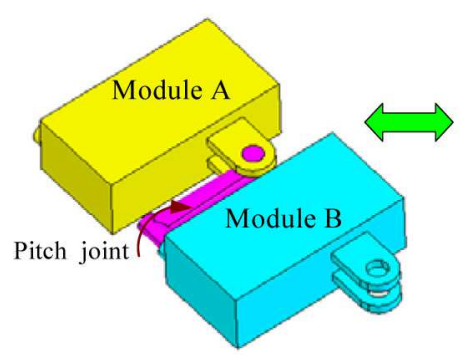

(e)

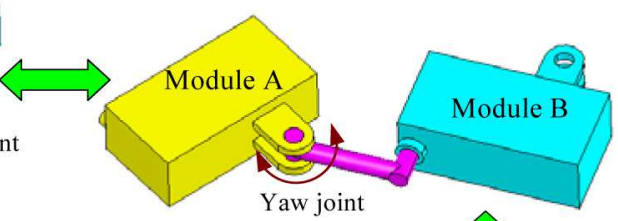

Yaw joint

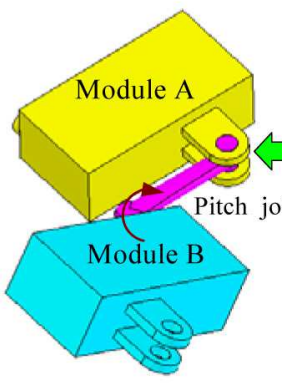

(d) (b)

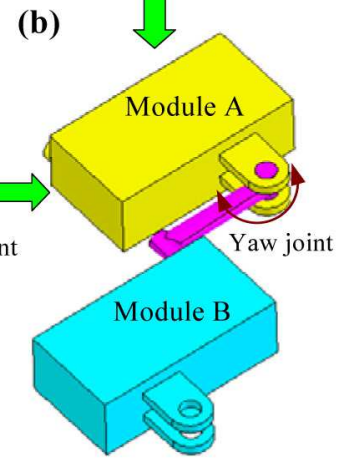

(c)

Fig. 2. Reconfiguration process of the proposed link-type structure

For two modules, there are three trim configurations shown in Figure 2 (a), Figure 2 (c) and Figure 2 (e), which are also called mobile configuration. The mobile configurations in this paper are defined to be the non-isomorphic configurations with parallel driving modules in the same plane. That is, in the mobile configuration, all the modules should contact the ground in parallel. In following sections, we merely take the mobile configurations into consideration in configuration analysis. Figure 3 (a) provides a top-view of these three mobile configurations. When designing the module and the linkage arm, the length of the module and the arm are strictly constrained to realize a trim figuration. The longitudinal dimension is designed in scale while the lateral dimension is free to some extent. The geometrical relationships in longitudinal direction shown in Figure 3 are given by

$$
l_{a}=l_{b}+l_{c}
$$

where $l_{a}$ is the length of the module body, $l_{b}$ is the length of the link arm, and $l_{c}$ is the distance between Yaw joint to the front or the back side of the module body as shown in Figure 3(a). 


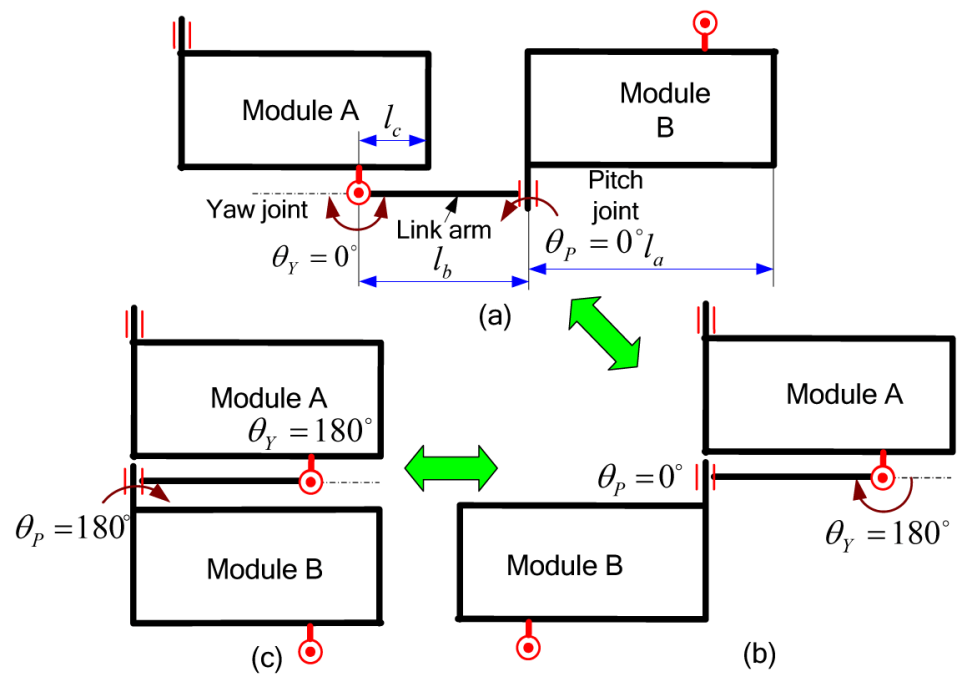

Fig. 3. Geometrical relationship of the link-type structures

\section{The prototype of AMOEBA-I}

\subsection{Mechanical structure of AMOEBA-I}

According to the principle mentioned above, a three-module robot AMOEBA-I has been developed. A single-module is mainly composed of a link arm, a track driving system, an offset Yaw joint driving system, a Pitch joint driving system. The module in Figure 4 is a standard one. There are often two or more wheels to support the track in the traditional track device. One of them is an active wheel and the others are passive. A modification is made on the wheel system as shown in Figure 4. First, we only use two wheels in the driving system. Second, the wheels are hollow inside. Such kind of wheel is light in mass. Moreover, it is possible for application of the system in water for its hollow body and lager volume. It has been taken into consideration that if the system is water proof, the robot can move both on land and in water like an amphibian vehicle. It has three DC motors which are for the track-driving, for pitch joint, and for yaw joint respectively. The motors are packed in the center box. Chain transmission device has been used in pitch joint and track's driving, while the yaw joint uses bevel gear pairs. Timing pulleys are used to driving the wheels forwards and backwards. The link arm and the link handle are used to connect and disconnect the adjacent modules. As shown in Figure 5, AMOEBA-I is repetitively composed of such kind module. It is a tracked robot which can overpass various terrains by transforming its configurations. The key advantage of this type over other link-type vehicle is its adaptability to environments through various configurations as shown in Figure 6. 

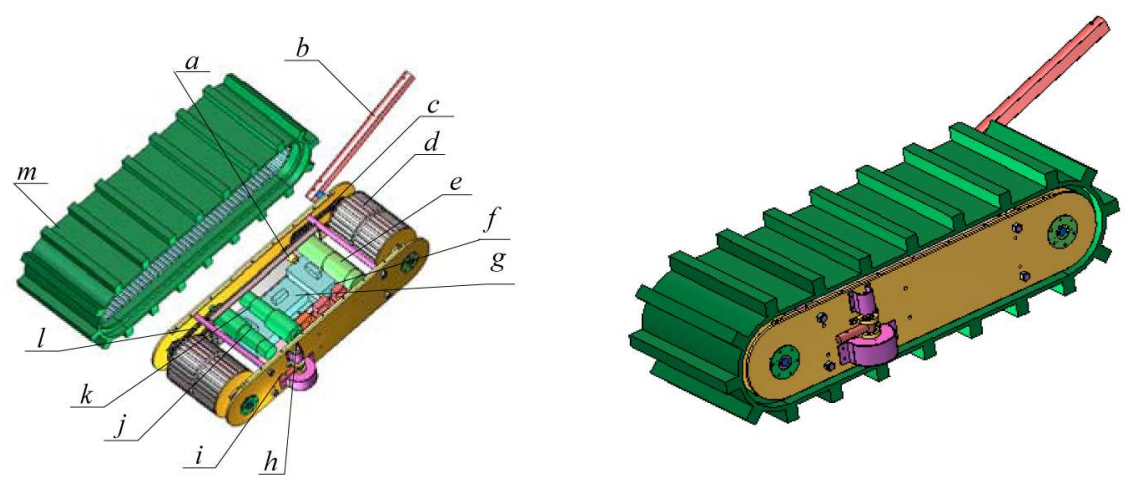

a. Pitch joint potentiometer; b. Link arm; c. Pitch joint chain driving; d. track wheel; e. Pitch joint motor; f. Motor controller; g. Motor drive; h. Yaw joint potentiometer; i. Yaw joint bevel gear driving; j. Track driving motor; $\mathrm{k}$. Yaw joint motor; 1 . Track wheel chain driving; $\mathrm{m}$. Track

Fig. 4. Structure of a single standard module of the prototype

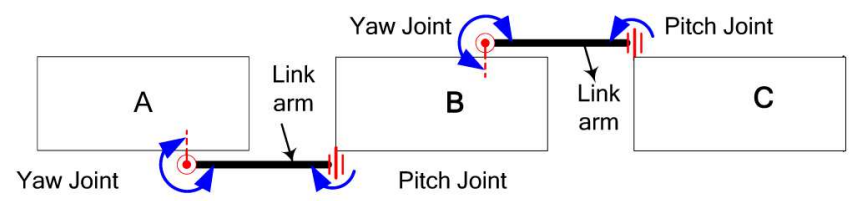

(a)
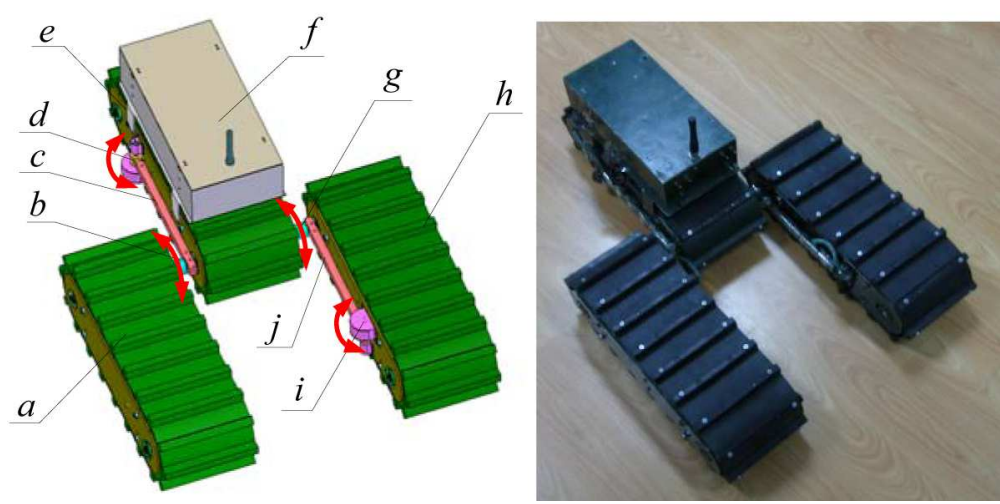

a. Module C; b. Pitch joint of Module C; c. Link arm between Module B and Module C; d. Yaw joint of Module B; e. Module B; f. Autonomous control system; g. Pitch joint of Module B; h. Module C; i. Yaw joint of Module A; j. Link arm between Module A and Module B. (b)

Fig. 5. Structure of AMOEBA-I 
AMOEBA-I has 9 kinds of available mobile configurations (Liu et al, 2007b). And it has three kinds of symmetry configurations: the line type (a), the triangle type (e) and the row type (i). The robot may change its configuration to adapt the various environment and tasks. Each configuration has various gaits and dimensions. The characteristics of AMOEBA-I can be generalized as:

(1) They have many non-isomorphic configurations. Their configuration can change automatically to adapt to the environment. With two or more modules, they can pose line type and row type easily. For instance they can pass the narrow space and the hole in line and they can move on uneven terrain safely or steering easily in row.

(2) It is small, lightweight and easy to carry. And it can resist against dust, gas and other hostile environment.

(3) With hollow wheels and sealed body, it can move in shallow water. The wheel has been designed to be hollow inside for underwater case.

(4) It is modularized and reconfigurable. The modular robot has satisfied maintenance and interchangeableness. It is reconfigurable in urgent need. The connection and disconnection of the modules can be finished through the link arm and the link handle.

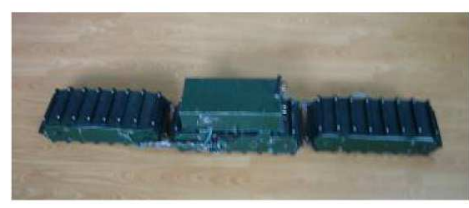

(a)

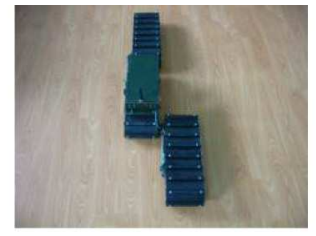

(b)

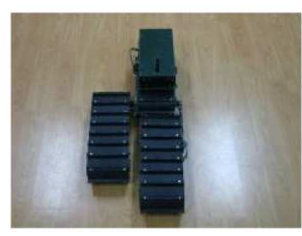

(c)

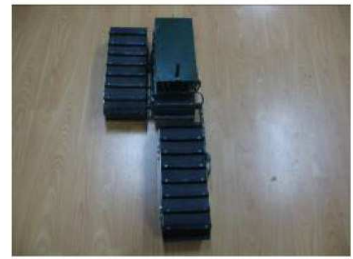

(d)

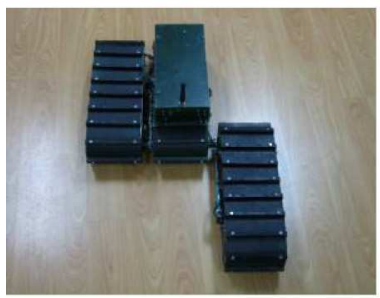

(g)

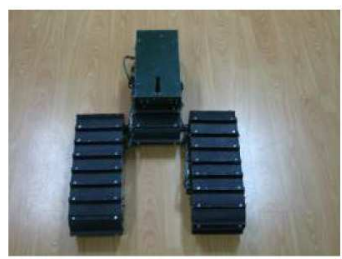

(e)

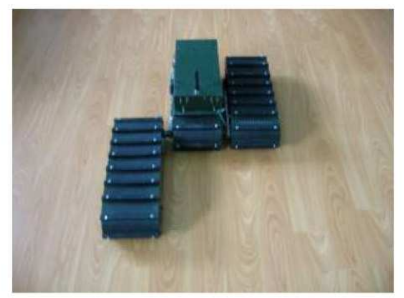

(h)

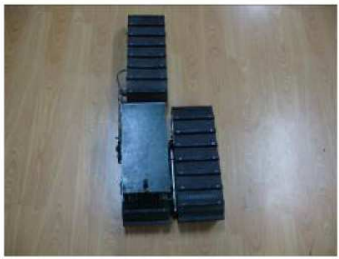

(f)

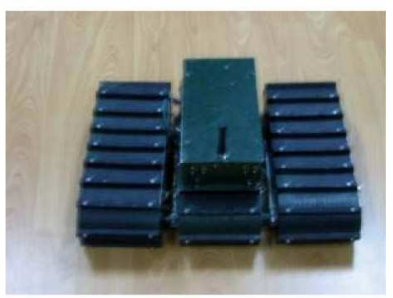

(i)

Fig. 6. Available configurations of AMOEBA-I 


\subsection{Structure of the control system}

The control system is composed of a wireless module, a main control module, motor control modules and sensor-based feedback control modules. It adopts PC-and-MCU structure as shown in Figure 7. Modularized structure has been widely used in the control system of mobile robots to enhance the error tolerance. As shown in Figure 7, the hardware of control system for a single module is modularized for improving the error tolerance and exchangeable ability of the system. The control system is composed of a supervisor system, an autonomous control system, and an actuator system. Each motor control unit of the actuator system has a microprocessor to deal with the information and control command of the motors and sensors. To exchange data among micro-controllers, the robot needs a simple and high efficient bus to serve as the system bus. CAN bus is a kind of shared broadcast field bus, in which all the nodes can send and receive message. Error detection, correction, and exchange have been implemented by the CAN controller. Thus CAN bus is selected as the suitable information bus in control system of AMOEBA-I. The CAN bus bridges the information among the three independent modules.

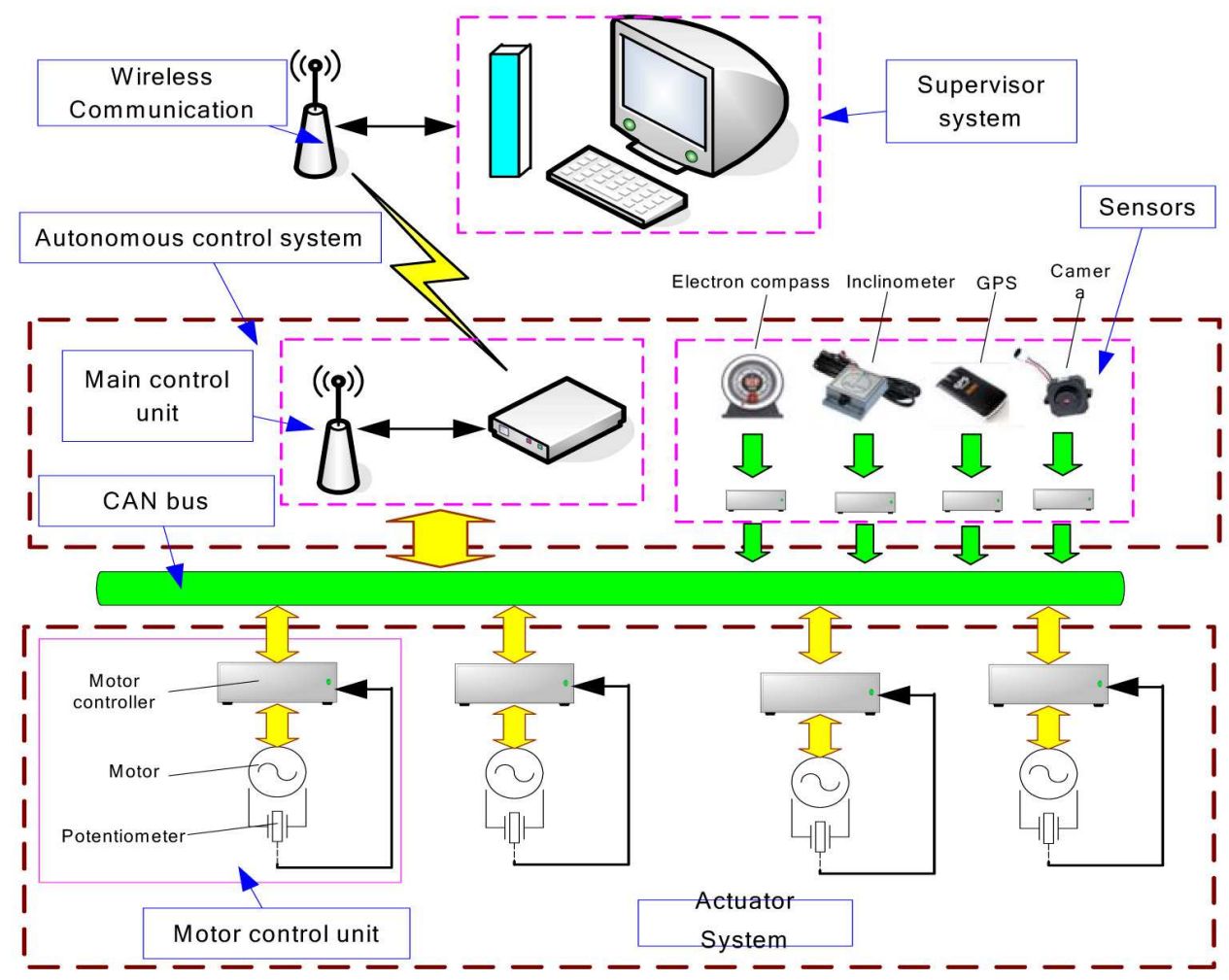

(a) Control system structure 


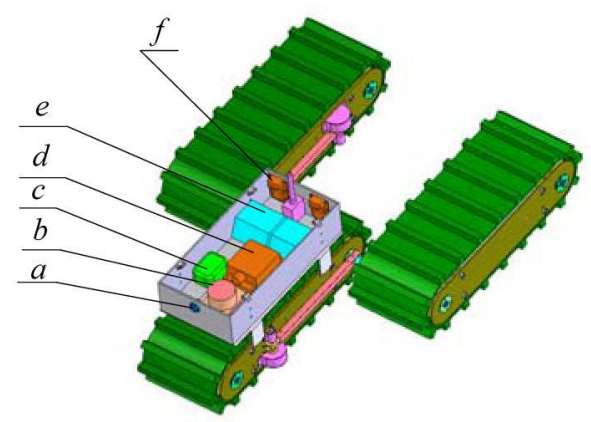

a. Camera; b. Electron compass; c. GPS; d. inclinometer; e. Power; f. Main control unit

(b) Details of the autonomous control system

Fig. 7. Control system of AMOEBA-I

Remote control and teleoperation control are required in a search and rescue robot for the real tasks since the disaster site is unreachable for the men. The supervisor system and main control unit in the control system communicate with each other through the wireless communication module. The supervisor system provides enough perception and command capabilities to the operator so that one can control the remote robot. The supervisor system not only sends the commands to the robot system, but also timely receives the status information returned by the robot, which include the configuration, joints angle, and the current consumed by the motor. The main unit is the kernel of the autonomous robot system. The main control unit is the decision-making unit of the robot, which estimates its own position, and status of the environment provided by the sensor-based feedback control unit and plans the movement of robot by using corresponding algorithms. Moreover, it is also used to globally program the movement of the robot and the change between the configurations. The main control unit has been designed and equipped in each mechanical module to implement its global modularization. It is also a communication interface between the robot and human-supervision platform by transmitting data from sensors to human-supervision platform through wireless communication module. The main control unit links all the dispersive motor control units, which are designed in the same method in order to implement the modularization of control system. It makes the whole system more extendable and stable. The main control unit and pinhole camera are placed in the control platform of middle module of AMOEBA-I. The motor control units implement the control for mechanical components in AMOEBA-I. All motor control units have the same structure, which receive the commands from the main control unit. After analysis and computation, actuators are set to a predefined position and rotational speed by using serial ports. When one module fails, it would be selected out of the whole control system to keep the system robust. The motor control unit is composed of the Fujitsu MCU, the CAN bus driver, the potentiometer, the DC motor and the DC controller. The motor control units collect each joint's angle form the A/D transformer in the MCU and then transfer it to main controller unit, which will help the main control unit plan the system globally.

The sensor system contains a camera, an inclinometer, an electron compass, and a GPS. It receives information from the environment and transmits the data to respective controllers in which pre-settled algorithms are used to deal with them, and then results are sent to the MCU. 


\section{A-B autonomy of AMOEBA-I}

\subsection{A-B autonomy with various configurations}

A-B autonomy or A-B mobility is the key autonomous capability for unmanned system (Army Science Board, 2002; Research Council of the National Academies Technology, 2002; Quek, 2005). It is also absolutely important for urban search and rescue robots. When in rescue operations, the robots are usually arranged to arrive a designated place to search the survivals and/or reconnoitre the environments. When in AMOEBA-I's A-B autonomy consideration, AMOEBA-I can overpass various terrains by transforming its configurations. Figure 8 provide a conceptual layout of AMOEBA-I's A-B autonomy with comparison of various configurations. Path $d$, Path $c$, and Path a denote the robot's best available route in Row configuration, Line configuration or Triangle configurations respectively. However, AMOEBA-I maybe have a most-best route Path $b$ with compound configurations which considered being shorter and/or safer.

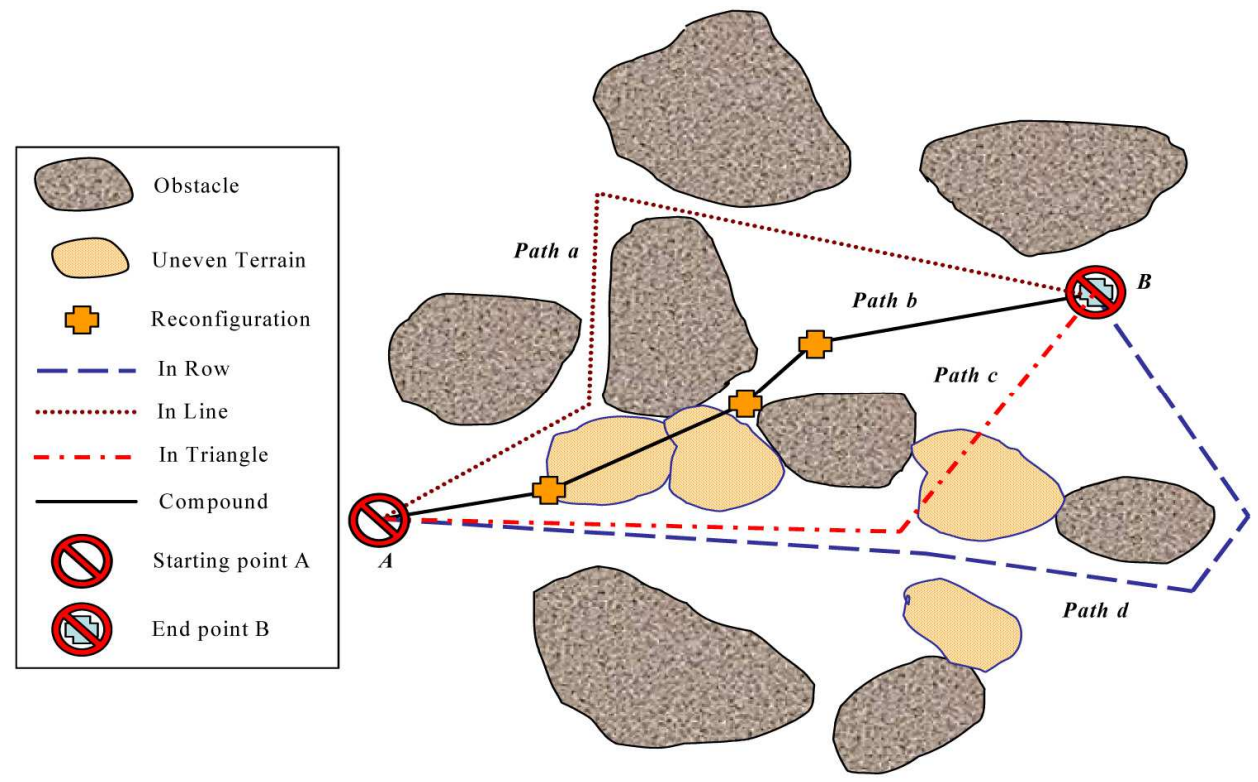

Fig. 8. A-B autonomy of AMOEBA-I with various configurations

When AMOEBA-I moves automatically in the unstructured environment, it must use the sensors to know the environment, and then select the optimal configuration according to the environment. So knowledge about the performance differences for various configurations and the rules of autonomous shape-shifting are very important for AMOEBA-I in its A-B autonomy. 


\subsection{Comparison of motion performances of AMOEBA-I}

In order to constitute the rules of autonomous shape-shifting for AMOEBA-I, we test the adaptability of AMOEBA-I in symmetrical configurations as shown in Figure 9.

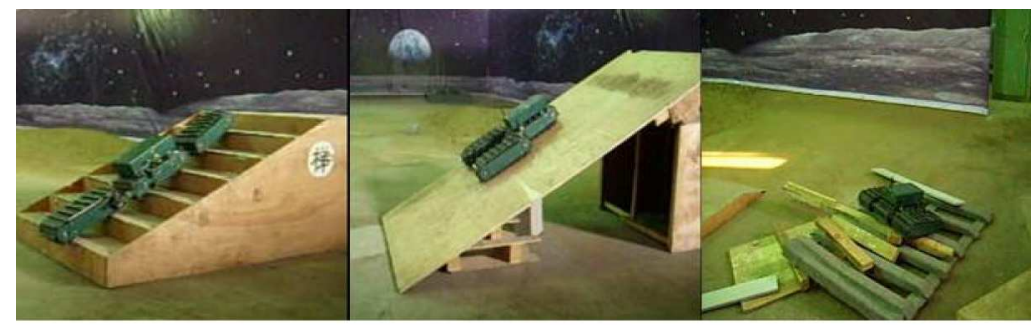

(a) Experiments under indoor environments

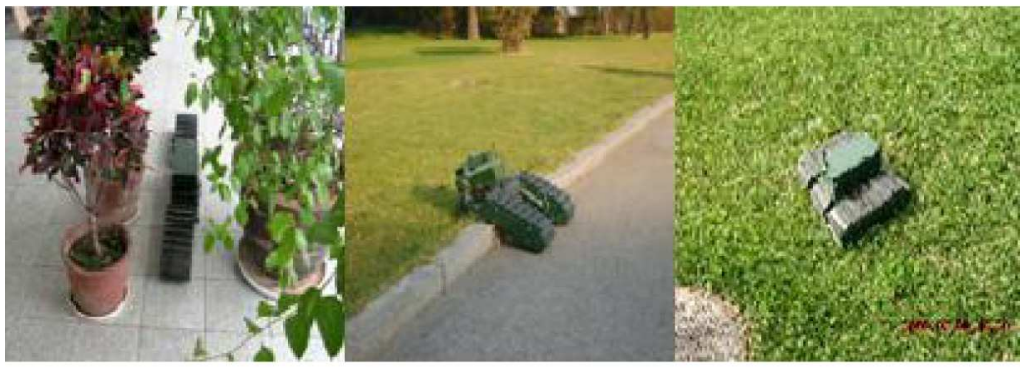

(b) Experiments under outdoor environments

Fig. 9. Experiments for the adaptability of AMOEBA-I

We have gotten the performances of AMOEBA-I by the experiment results as shown in Table 1.

\begin{tabular}{|c|c|c|c|}
\hline Environment type & $\begin{array}{c}\text { Line } \\
\text { configuration }\end{array}$ & $\begin{array}{c}\text { Triangle } \\
\text { configuration }\end{array}$ & $\begin{array}{c}\text { Row } \\
\text { configuration }\end{array}$ \\
\hline Uneven outdoors ground & II & I & III \\
\hline Obstacles & I & II & III \\
\hline Slopes & I & II & III \\
\hline Stairs & I & II & III \\
\hline Flat ground & III & II & I \\
\hline Turning & III & II & I \\
\hline Long and narrow spaces & I & III & II \\
\hline
\end{tabular}

Table 1. Motion performances of three symmetrical configurations

In Table 1, "I", "II" and "III" stand for three levels as "good", "common" and "poor", respectively. From Table II we can get conclusions as follows: The robot can climb up obstacles with satisfying performance as well as get across cabined spaces under Line 
configuration, which is a remarkable improvement for environment-adaptation. In Triangle configuration, stability and the mobility of climbing slopes are very notable. Zero-radius turning can be realized easily under Row configuration which makes it be more agilely and be minimum energy consumed.

\subsection{Rules of autonomous shape-shifting in A-B autonomy}

Since the robot mostly moves on flat ground, we choose Row configuration as the initial configuration. The robot takes the following methods to recognize different environments and adopts the most appropriate configuration. Here we will take the field, obstacle and slopes as examples respectively to show our rules of how to guide the robot to change its configuration.

(1) Environments of uneven ground in the field. In this situation, data from the inclinometer are taken as the most important into consideration. If the obliquity does not exceed the threshold of 10 degrees, we define the ground as general uneven ground. The states fall into 2 kinds according to the specific feedback from inclinometer.

a. The slope's gradient changes not fast (if mean square errors are smaller than 20 with 10 times' continuously samplings), then the ground is thought to be flat and Row configuration is taken.

b. The slope's gradient changes rapidly (if mean square errors exceed 20 with 10 times' continuously samplings), then the ground is thought to be uneven, and the robot takes the Triangle configuration.

(2) Environments of obstacles. When obstacles are in its way, the robot is in one of the three following configurations. Configuration changing is decided upon the feedback information from encoder and the motor currents.

a. Row configuration. If data from motor encoder does not change in 10 seconds, and electric current value steps to a high level, we can conclude that the robot has some obstacles in its way and it cannot get over, so the robot changes into Triangle configuration. Otherwise, keep in current configuration and move on.

b. Triangle configuration. If data from encoder does not change in 10 seconds, and current value steps to a high level, we can conclude that the robot meets some large obstacles hard to pass. In such case, the configuration alters into Line form. Otherwise, keep in current configuration and move on.

c. Line configuration. If data from encoder does not change in 10 seconds and current value steps to a high level, which means large obstacles are in the way and the robot has to avoid them with utilizing information from electron compass and encoder. Otherwise, keep in current configuration and move on.

(3) Environments of slopes. Information from the inclinometer is at the first place to be considered when the robot gets over obstacles with certain gradients. The strategy of its locomotion is illuminated as:

a. The gradients range in $\left[0,10^{\circ}\right]$ : In this situation, there is no need to alter the robot's configuration. 
b. The gradients range in $\left[10^{\circ}, 20^{\circ}\right]$ : Triangle configuration which is prone to get over obstacles and has good stability is available.

c. The gradients range in $\left[20^{\circ}, 30^{\circ}\right]$ : Take Line configuration for the robot may overcome tough obstacles in this kind.

d. The gradients exceed $30^{\circ}$ : Steer clear of the slopes using data from electron compass and encoder.

In a word, the robot can judge its surroundings accurately by analyzing data from sensors. Moreover, it can take different configurations to adapt to the environment and complete the designated mission.

\subsection{GPS based autonomous navigation}

We used GPS to provide geographic coordinates. Some calculation should be done to get the robot's present position and the difference between its present and target position. Set these differences as the norm angles of electron compass, the robot will move towards target position. As is shown in Figure 10, $\mathrm{O}$ is set to be the mobile robot's initial position, whose geodetic coordinates are $\left(L_{0}, B_{0}\right)$, and $\mathrm{P}$ is the target position with its geodetic coordinates at $\left(L_{P}, B_{P}\right)$. The dashed circle is the confines of the range in which the robot may stop at the range of allowable error. After the robot covers a certain distance, it stops at $A\left(L_{A}, B_{A}\right)$ to adjust its orientation. As it passed $B\left(L_{B}, B_{B}\right)$, it enters GPS blind zone. At $C$, the robot makes its orientation adjustment utilizing geodetic coordinates according to information of inertial components. The robot leaves GPS blind zone from the moment it gets to $\mathrm{D}\left(L_{D}, B_{D}\right)$. Suppose it meets an obstacle at E, the navigation program will stop and a program of obstacle-get-over or obstacle-avoidance will take charge until it gets over or passes by the obstacle. The robot changes its configuration at $F$ and alters into its former shape at $G\left(L_{G}\right.$, $\left.B_{G}\right)$. In this graph, $\mathrm{H}\left(L_{H}, B_{H}\right)$ is its end point.

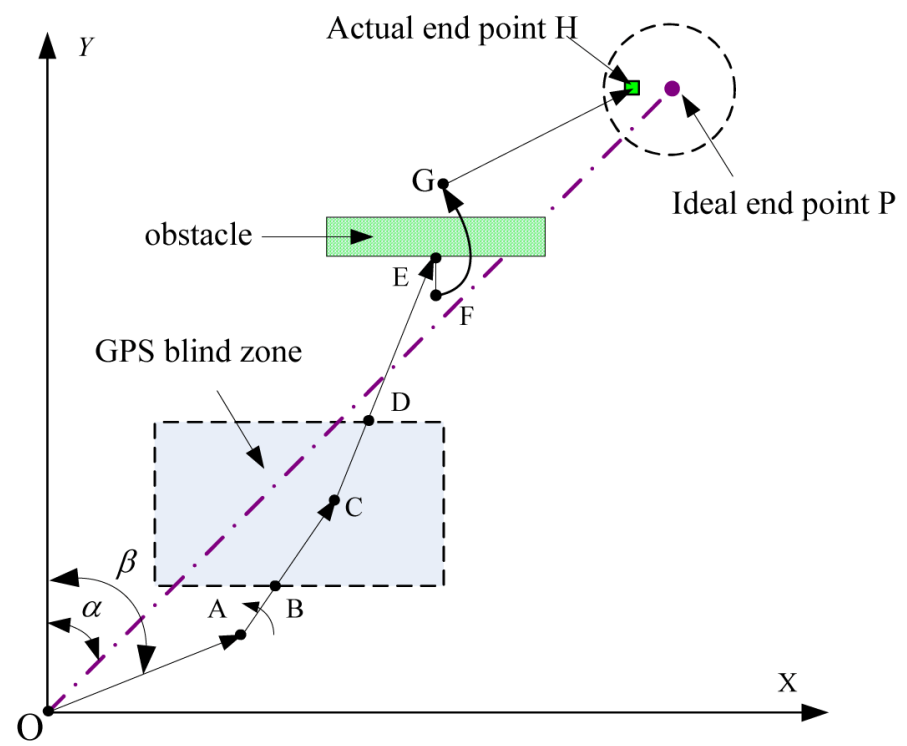

Fig. 10. Autonomous navigation of AMOEBA-I 
After we select an end point, $\mathrm{OP}^{\prime}$ s direction represented by a can be ascertained.

$$
\alpha=\frac{L_{P}-L_{0}}{B_{P}-B_{0}} \cos B_{0}
$$

The electron compass gives the robot's deflexion angle $Y$ (the angle to $Y$ axis) at its initial position. Therefore, at the beginning, the robot has to turn $\theta$, which can be calculated by

$$
\theta=\alpha-\gamma
$$

If $\theta>0$, it turns left, while it turns right if $\theta<0$, otherwise it goes straightforward. Suppose the robot reaches a where GPS fails after certain periods (the interval between the robot samples the GPS values), it starts to calculate its position and orientation using the record of inertia components.

After one period, the robot moves to B, whose geodetic coordinates can be calculated by

$$
\begin{aligned}
& B_{B}=B_{A}+\frac{v t \cos \gamma}{K_{1}} \\
& L_{B}=L_{A}+\frac{v t \sin \gamma}{K_{2}}
\end{aligned}
$$

In Eqs. (3) and (4), $v$ is the velocity of robot, $\gamma$ represents its including angle to $\mathrm{Y}$ axis and $t$ is its motion period. $K_{1}$ is chosen to be $30.8 \mathrm{~m} / \mathrm{s}$, which is the distance between the adjacent latitude divided by minutes along the same longitude.

$$
K_{2}=K_{1} \cos B_{A}
$$

where $K_{2}$ in Eq.(5) denotes the distance along BA latitude between every one minute longitudes. Whether it turns left or right can be concluded by Eqs. (2) and (3). Therefore, even if GPS system cannot receive the satellites' signals, orientation program by inertia components is automatically performed, in which data from the encoder, electron compass and inclinometer together with the previous GPS's information are used to conclude the robot's present geodetic coordinates to make sure its navigation works correctly. When it gets out of the blind zone, GPS system starts to work for the robot's orientation. E-F-G is the path followed by the robot when it gets over the obstacles after shape-shifting or round the obstacle directly. In this process, the robot checks the obstacles first. If the obstacle is too tough to get over, obstacle-avoidance program runs to make the robot pass by the obstacle. If it founds that the obstacle can be got over after shape-shifting, it will withdraw a certain distance and change into a more appropriate configuration. After getting over the obstacle, the robot will take a more efficient configuration, reorient itself and move towards the target.

In addition, the robot checks whether it reaches the target position every period by calculating the distance between its position and the target. Suppose that the robot's present position is $C\left(L_{C}, B_{C}\right)$, then the distance to the target position $\mathrm{P}$ can be gotten by 


$$
L=\sqrt{\left(K_{1}\left(B_{P}-B_{C}\right)\right)^{2}+\left(K_{2}\left(L_{P}-L_{C}\right)\right)^{2}}
$$

As a measurement error lies in GPS system, the distance from P to C signed as L cannot be simply used to decide whether the robot has reached its target. If L's error lies at a tolerable range, the robot is thought to be get to the aim point; If not, then we let $C$ replace $B$, recalculate $a$ and do the conclusion and analysis that has been illuminated in the above paragraphs until we get an $\mathrm{L}$ that meets the error tolerance.

\section{Autonomous motion experiments}

Autonomous navigation experiments free of obstacles as well as experiments in the environment where the robot has to reshape itself to get over the small obstacles have been made with AMOEBA-I.

\subsection{Experiment system and experimental field}

The experiment system include one console and one AMOEBA-I. The experiment system and Experimental field to validate the autonomous navigation and shape-shifting algorithm are shown in Figure 11.

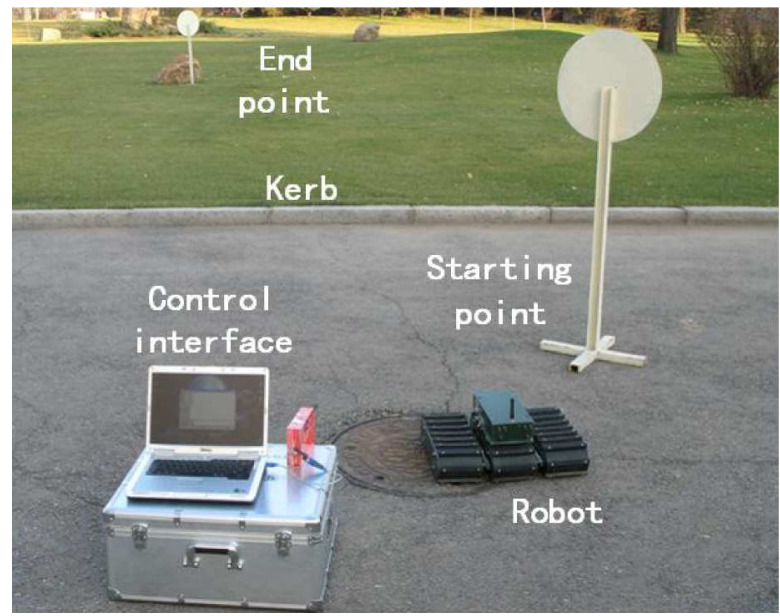

Fig. 11. Experiment system

\subsection{Autonomous motion in the no-obstacle situation}

AMOEBA-I has moved automatically from starting point to end point on the lawn. There is no-obstacle situation in the way that AMOEBA-I move on. Process of autonomous motion is shown in Figure 12. 


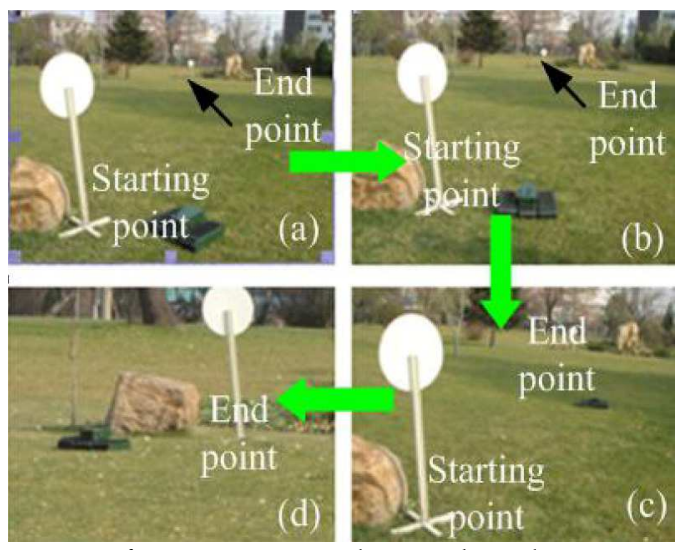

Fig. 12. Autonomous motion of AMOEBA-I in the no-obstacle situation

In the experiment, we set the target position at $41^{\circ} 45.6950^{\prime} \mathrm{N} / 123^{\circ} 26.5642^{\prime} \mathrm{E}$.

Figure 12 (a) shows the robot's orientation before the aim point was set, with its position at $41^{\circ} 45.6945^{\prime} \mathrm{N} / 3^{\circ} 26.5345^{\prime} \mathrm{E}$ measured by GPS devices and the electron compass read 135 degrees.

Figure 12 (b) shows that after the aim point were set, the robot adjusted itself to face the target.

Figure 12 (c) shows one of the several adjustments on the way caused by the errors of GPS $(<15 \mathrm{~m})$ and electron compass $(-30 \sim 30)$.

Figure $12(\mathrm{~d})$ shows the robot stopped near the target at $41^{\circ} 45.6948^{\prime} \mathrm{N} / 123^{\circ} 26.5340^{\prime} \mathrm{E}$.

\subsection{Autonomous motion in the small obstacle situation}

The process of robot's autonomous navigation and autonomous shape-shifting is shown in Figure 13. Each step's motion is introduced as follows.

Figure 13(a): AMOEBA-I is located at the starting point.

Figure 13(b): After climbing obstacles, AMOEBA-I detected that Row configuration cannot cross.

Figure 13(c) and Figure 13(d): it returns and changes into the Triangle configuration with stronger ability to cross the obstacle.

Figure 13(e): The process of AMOEBA-I with Triangle configuration cross the obstacle.

Figure 13(f), Figure 13(g) and Figure 13(h): AMOEBA-I detected that the ground slope $<10^{\circ}$, it turn back into Row configuration with good turning ability.

Figure 13(i): The point at which AMOEBA-I adjust its orientation.

Figure 13(j): AMOEBA-I arrives at target point and completes the process of autonomous navigation.

As the experimental results show, in the process of autonomous motion, when AMOEBA-I encountered a vertical obstruction or obstacle with small slope and angle, it can make a reaction in time and automatically change into other configuration with strong ability to climb up obstacle directly and approximate the target point. 


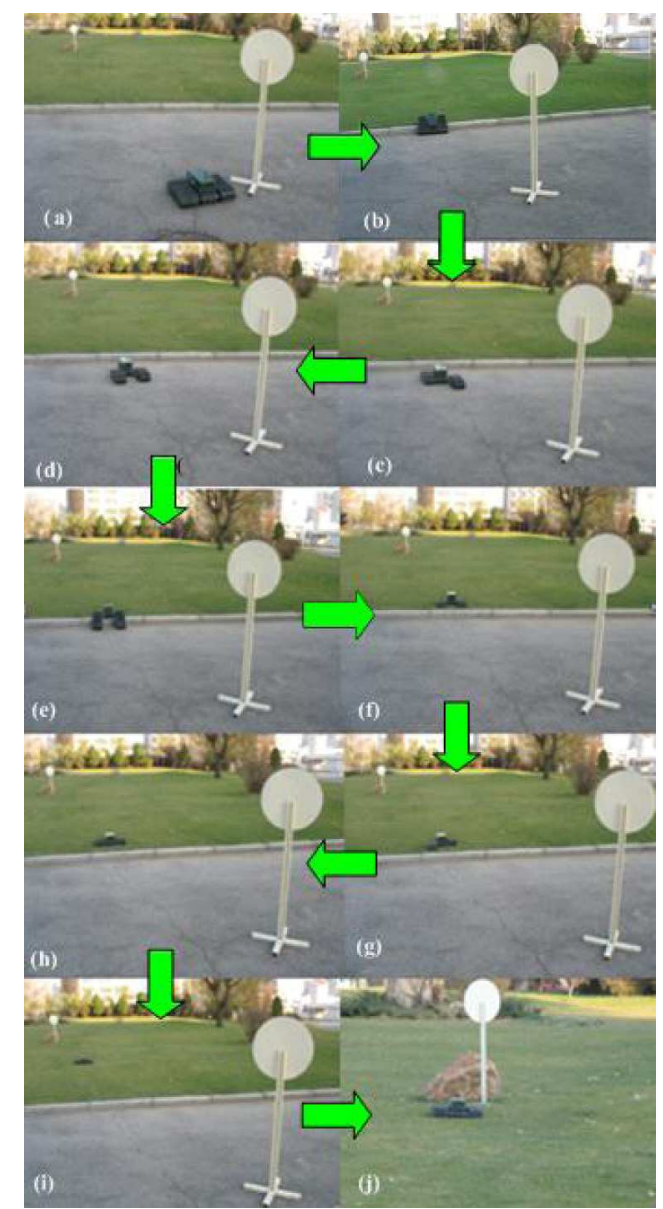

Fig. 13. Autonomous motion of AMOEBA-I in the obstacle situation

\section{Conclusion}

The frequent nature disasters and man-made catastrophes during the last decades such as earthquake, typhoon, hurricane, radiation and terrorist attack have greatly aroused people's attention on the importance of Urban Search and Rescue (USAR). In some extremely dangerous catastrophes, the rescue staffs are eager to know the inner situation while they cannot perform an in-depth reconnaissance. Robots are emergently needed for tactical search and rescue, which covers how the field teams actually find, support, and extract survivors. AMOEBA-I, a three-module shape-shifting robot with nine configurations from Shenyang Institute of Automation (SIA), Chinese Academy of Sciences (CAS), has been developed for such cases. In this research we mainly focused on the A-B autonomy of AMOEBA-I. With global considerations of the shape-shifting robot and its locomotion environments, this chapter presented a multi-sensor-based autonomous control system for the robot "AMOEBA-I" to move outdoors. With the advantages of various configurations, 
AMOEBA-I has high flexibility under unstructured environments. When AMOEBA-I moves in autonomous mode, it can use its sensors to know about the environment, and then select the optimal configuration to achieve an A-B autonomy mission. Experiments have been mainly executed to validate the effectiveness of the system. Since the urban search and rescue environments are usually unstructured and unpredictable, we believe that the shape changing should be an ideal solution to improve the rescue robot's mobility, flexibility, and adaptability. This research result provides a fundamental approach for robot's possible operation in more complex environments.

\section{Acknowledgement}

This research is supported partly by the National Natural Science Foundation of China (Grant No. 60705029), the National High-Technology 863 Program (Grant Nos. 2006AA04Z254, 2007AA041502-5), Doctoral Startup Foundation of Liaoning Province and State Key Laboratory of Robotics foundation (Grant No. RLO200813).

\section{References}

Army Science Board (2002). Ad Hoc Study on Human Robot Interface Issues: Final Report, Virginia Blackburn, M.; Bailey, R. \& Lytle, B. (2004). Improved mobility in a multi-degree-of-freedom unmanned ground vehicle. Proceedings of SPIE, Vol. 5422, pp. 124-134

Casper, J. \& Murphy, R. (2003). Human-robot interaction during the robot-assisted urban search and rescue response at the world trade center. IEEE Transaction on System, Man, and Cybernetics, Part B: Cybernetics, Vol. 33, No. 3, pp. 367-383

Erkmen, I.; Erkmen, A. M.; Matsuno, F.; Chatterjee, R. \& Kamegawa T. (2002). Snake robots to the rescue. IEEE Robotics \& Automation Magazine, Vol. 9, No. 3, pp. 17-25

Hirose, S \& Fukushima, E. (2002). Development of mobile robots for rescue operations. Advanced Robotics, Vol. 16, No. 6, pp. 509-112

Huang, B.; Li, M. \& Sun, L.(2006) The research of a parent-children type robot system. Proceedings of IEEE International Conference on Robotics and Biomimetics, pp. 977-981

Li, B.; Ma S.; Liu, J. \& Wang, Y. (2006). AMOEBA-I: an improved link-type shape shifting modular robot for search and rescue operation. Proceedings of IEEE International Workshop on Safety, Security, and Rescue Robotics

Lim, S.; Park, D.; Kwak, Y.; Kim, B. \& Jeon, S. (2005). Variable geometry single-tracked mechanism for a rescue robot. Proceedings of IEEE International Workshop on Safety, Security, and Rescue Robotics. pp. 111-115

Liu, J.; Wang, Y.; Li, B. \& Ma S. (2004). Link-type shape shifting modular robot for search and rescue. High Technology Letters, Vol. 10, sup, pp. 179-183

Liu, J.; Wang, Y.; Li, B. \& Ma S. (2007a). Current research, key performance and future development of search and rescue robot. Frontiers of Mechanical Engineering in China, Vol. 2, No. 4, pp. 404-416

Liu, J.; Wang, Y.; Li, B.; Ma S. \& Tan, D. (2007b). Center-configuration selection technique for the reconfigurable modular robot. Science in China Series F-Information Sciences, Vol. 50, No. 5, pp. $697-710$ 
Matsuno, F. \& Tadokoro, S. (2004). Rescue Robots and Systems in Japan. Proceedings of IEEE International Conference on Robotics and Biomimetics, pp. 12-20

Murphy, R. (2002). Marsupial and shape-shifting robots for urban search and rescue. IEEE Intelligent Systems, Vol. 20, No. 3/4, pp. 14-20

Murphy, R.; Tadokoro, S.; Nardi, D.; Jacoff, A.; Fiorini, P.; Choset, H. \& Erkmen, A. (2007). Search and Rescue Robotics, in: Springer Handbook of Robotics, B. Siciliano et al. (Ed.), pp. 1151-1173, Springer Berlin Heidelberg, Berlin

Osuka, K. \& Kitajima, H. (2003). Development of Mobile Inspection Robot for Rescue Activities: MOIRA. Proceedings of IEEE/RSJ International Conference on Intelligent Robots and Systems, pp. 3373-3377

Quek, B.K.; Ibanez-Guzman, J. \& Lim, K.W. (2005). Feature-based perception for autonomous unmanned navigation. Proceedings of $31^{\text {st }}$ Annual Conference of IEEE Industrial Electronics IECON, pp. 1791-1796

Research Council of the National Academies Technology (2002). Development for Army Unmanned Ground Vehicles, The National Academic Press, Washington D.C.

Tabata, K.; Inaba, A. \& Amano, H. (2005). Development of a transformational mobile robot to search victims under debris and rubble -2nd report: improvement of mechanism and interface. Proceedings of IEEE International Workshop on Safety, Security, and Rescue Robotics. pp. 19-24

Tetsushi, K.; Yamasaki, T.; Igarashi, H. \& Matsuno, F. (2004). Development of the snake-like rescue robot "KOHGA". Proceedings of IEEE Conference on Robotics and Automation, pp. 5081-5086

Wolf, A.; Brown, H.; Casciola, R.; Costa, A.; Schwerin, M.; Shamas, E. \& Choset, H. (2003). A mobile hyper redundant mechanism for search and rescue tasks. Proceedings of IEEE/RSJ International Conference on Intelligent Robots and Systems, pp. 2889-2895

Zhang, H.; Wang, W.; Den, Z.; Zong, G \& Zhang, J. (2006). A novel reconfigurable robot for urban search and rescue. International Journal of Advanced Robotic Systems, Vol. 3, No. 4, pp. 359-366

Zou, D.; Wang, T. \& Liang, J. (2006). Reconfiguration research on modular mobile robot. Proceedings of IEEE/RSJ International Conference on Intelligent Robots and Systems, pp. 1082-1085 


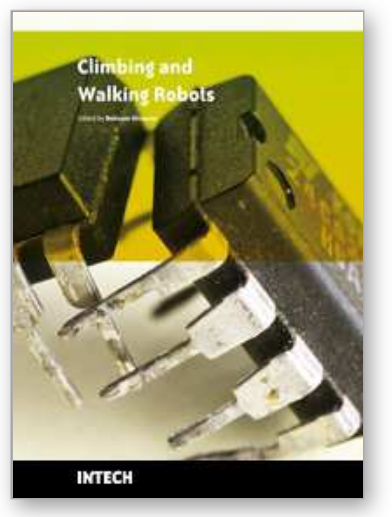

\author{
Climbing and Walking Robots \\ Edited by Behnam Miripour
}

ISBN 978-953-307-030-8

Hard cover, 508 pages

Publisher InTech

Published online 01, March, 2010

Published in print edition March, 2010

Nowadays robotics is one of the most dynamic fields of scientific researches. The shift of robotics researches from manufacturing to services applications is clear. During the last decades interest in studying climbing and walking robots has been increased. This increasing interest has been in many areas that most important ones of them are: mechanics, electronics, medical engineering, cybernetics, controls, and computers. Today's climbing and walking robots are a combination of manipulative, perceptive, communicative, and cognitive abilities and they are capable of performing many tasks in industrial and non- industrial environments.

Surveillance, planetary exploration, emergence rescue operations, reconnaissance, petrochemical applications, construction, entertainment, personal services, intervention in severe environments, transportation, medical and etc are some applications from a very diverse application fields of climbing and walking robots. By great progress in this area of robotics it is anticipated that next generation climbing and walking robots will enhance lives and will change the way the human works, thinks and makes decisions. This book presents the state of the art achievments, recent developments, applications and future challenges of climbing and walking robots. These are presented in 24 chapters by authors throughtot the world The book serves as a reference especially for the researchers who are interested in mobile robots. It also is useful for industrial engineers and graduate students in advanced study.

\title{
How to reference
}

In order to correctly reference this scholarly work, feel free to copy and paste the following:

Yuechao Wang, Jinguo Liu and Bin Li (2010). A-B Autonomy of a Shape-shifting Robot 'AMOEBA-I' for USAR, Climbing and Walking Robots, Behnam Miripour (Ed.), ISBN: 978-953-307-030-8, InTech, Available from: http://www.intechopen.com/books/climbing-and-walking-robots/a-b-autonomy-of-a-shape-shifting-robotamoeba-i-for-usar

\section{INTECH}

open science | open minds

\section{InTech Europe}

University Campus STeP Ri

Slavka Krautzeka 83/A

51000 Rijeka, Croatia

Phone: +385 (51) 770447

Fax: +385 (51) 686166

\section{InTech China}

Unit 405, Office Block, Hotel Equatorial Shanghai

No.65, Yan An Road (West), Shanghai, 200040, China 中国上海市延安西路65号上海国际贵都大饭店办公楼 405 单元

Phone: +86-21-62489820

Fax: +86-21-62489821 
www.intechopen.com 
(C) 2010 The Author(s). Licensee IntechOpen. This chapter is distributed under the terms of the Creative Commons Attribution-NonCommercialShareAlike-3.0 License, which permits use, distribution and reproduction for non-commercial purposes, provided the original is properly cited and derivative works building on this content are distributed under the same license. 